\title{
Center-specific differences in mortality: Preliminary analyses using the Risk Adjustment in Congenital Heart Surgery (RACHS-1) method
}

Kathy J. Jenkins, MD, MPH

Kimberlee Gauvreau, ScD

From the Department of Cardiology, Children's Hospital, Boston, Mass.

This work was supported by National Institutes of Health/National Heart, Lung, and Blood Institute grant K08HL2936-01 (Dr Jenkins) and by the Kobren Fund (Dr Gauvreau).

Received for publication March 15, 2001; revisions requested Aug 17, 2001; revisions received Nov 2, 2001; accepted for publication Dec 14, 2001.

Address for reprints: Kathy J. Jenkins, MD, MPH, Children's Hospital, Department of Cardiology, 300 Longwood Ave, Boston, MA 02115 (E-mail: jenkins@ cardio.tch. harvard.edu).

J Thorac Cardiovasc Surg 2002;124:97-104

Copyright (C) 2002 by The American Association for Thoracic Surgery

0022-5223/2002 $\$ 35.00+0 \quad \mathbf{1 2 / 1 / 1 2 2 3 1 1}$

doi:10.1067/mtc.2002.122311
Objective: We sought to explore the usefulness of the Risk Adjustment in Congenital Heart Surgery method (designated RACHS-1) of adjusting for case-mix differences when comparing institutional mortality after surgery for congenital heart disease.

Methods: By using 1996 hospital discharge data from 6 states, centers performing at least 100 operations for congenital heart disease (patient age $<18$ years) were identified. Using the RACHS-1 method, procedures were grouped into 6 risk categories, and institutions were ranked in order of increasing mortality rate. A graphic display of ranks by risk category identified patterns of performance. Incorporating age, prematurity, and presence of a major noncardiac structural anomaly into multivariate models allowed computation of an overall risk-adjusted rank for each institution on the basis of its standardized mortality ratio.

Results: Among 109 centers performing 7177 operations for congenital heart disease, 22 performed at least 100 cases $(72.3 \%$ of total operations). Unadjusted mortality rates ranged from $2.5 \%$ to $11.4 \%$. A total of 4318 cases could be placed into 1 of the 6 risk categories. Few deaths occurred in risk category 1, and few institutions performed procedures in risk categories 5 and 6, making institutional comparisons in these categories uninformative. Considering mortality rates in categories 2 through 4, institutions displayed either relatively consistent ranks, a threshold increase in mortality as higher-risk procedures were performed, or a threshold decrease in mortality. Standardized mortality ratios indicated which institutions performed better or worse than expected on the basis of their case mix.

Conclusions: The RACHS-1 method can be used to judge relative institutional performance, either by evaluating within-risk-category differences or by comparisons of observed and expected mortality rates.

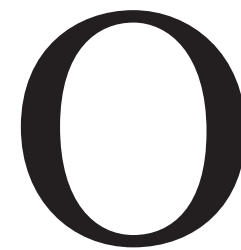

perations for congenital heart disease carry a significant risk of death for all but the simplest lesions. We and others ${ }^{1-7}$ have demonstrated considerable differences in mortality among institutions performing cardiac surgery in children. For individual centers, quality-improvement efforts can be stimulated by information about how one center is performing relative to others. Simple comparisons of overall mortality rates are not useful because of baseline differences in risk among individuals. Risk adjustment for adult cardiac procedures is easier than it is for pediatric procedures because there is less variation in the anatomy and physiology. For pediatric cardiac surgery, case-mix differences between institutions lie in the marked anatomic variation of the underlying disease and thus in the nature of the surgical procedure required for correction. Lesion-specific approaches have limited precision because even common lesions represent only a small fraction of the annual surgical caseload for a center. Despite its importance, 


\section{Table 1. Individual procedures by risk category}

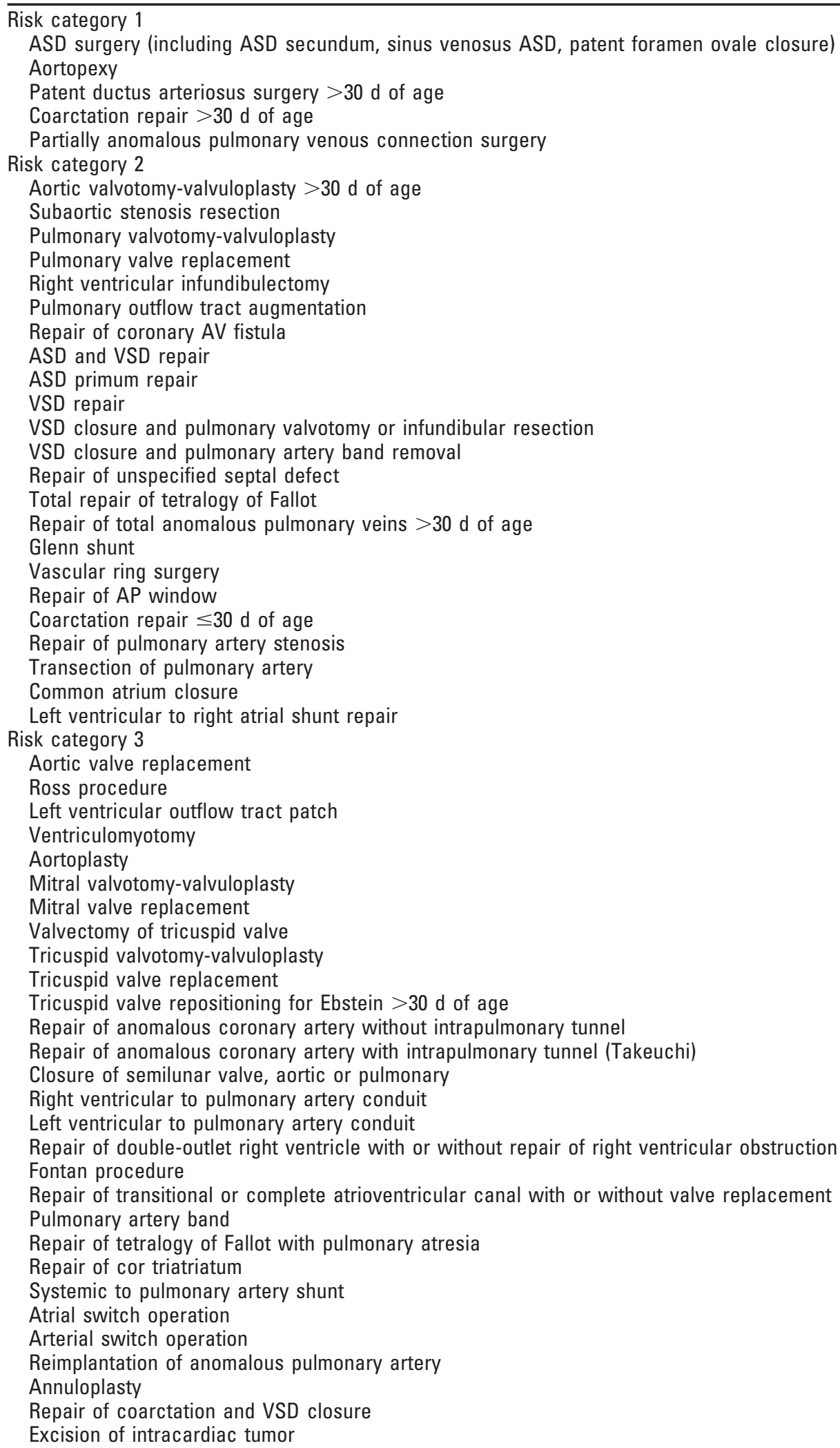




\section{Table 1-Continued}

Risk category 4
Aortic valvotomy-valvuloplasty $\leq 30 \mathrm{~d}$ of age
Konno procedure
Repair of complex anomaly (single ventricle) by VSD enlargement
Repair of total anomalous pulmonary veins $\leq 30 \mathrm{~d}$ of age
Atrial septectomy
Repair of transposition-VSD-sub PS (Rastelli)
Atrial switch operation with VSD closure
Atrial switch operation with repair of sub PS
Arterial switch operation with pulmonary artery band removal
Arterial switch operation with VSD closure
Arterial switch operation with repair of sub PS
Repair of truncus arteriosus
Repair of hypoplastic or interrupted arch without VSD closure
Repair of hypoplastic or interrupted aortic arch with VSD closure
Transverse arch graft
Unifocalization for tetralogy of Fallot-pulmonary atresia
Double switch
Risk category 5
Tricuspid valve repositioning for neonatal Ebstein $\leq 30 \mathrm{~d}$ of age
Repair of truncus arteriosus and interrupted arch
Risk category 6
Stage 1 repair of hypoplastic left heart syndrome (Norwood operation)
Stage 1 repair of nonhypoplastic left heart syndrome conditions
Damus-Kaye-Stansel procedure

$A S D$, Atrial septal defect; $A V$, atrioventricular; $V S D$, ventricular septal defect; $A P$, aortopulmonary; sub $P S$, subpulmonic stenosis.

estimating overall institutional performance for pediatric cardiac surgical centers can be challenging.

We have recently developed a consensus-based method to adjust for case-mix differences when comparing in-hospital mortality for groups of children undergoing surgery for congenital heart disease. ${ }^{8}$ Our method can provide reasonable estimates of overall institutional performance with fairly modest data requirements. To explore the usefulness of this new research tool, we analyzed hospital discharge data from centers with large surgical volumes in 6 states using the Risk Adjustment in Congenital Heart Surgery (RACHS-1) method. Our analyses uncovered several distinct patterns of programmatic outcomes, which should assist in program-specific efforts to reduce mortality.

\section{Methods}

\section{Data Sources}

We obtained and analyzed 1996 hospital discharge data from 6 states (California, Illinois, Massachusetts, New York, Pennsylvania, and Washington). These data sets contain information for each hospital discharge occurring within the year in standard formats. Up to 10 ICD-9-CM ${ }^{9}$ procedure codes and 25 diagnosis codes were available within each data set, along with additional patient and billing information. By using previously described algorithms, we selected abstracts from children less than 18 years of age with codes indicating surgical repair of a congenital heart defect. ${ }^{8}$ Cases with codes for cardiac transplantation or interventional catheterization procedures were eliminated, as were cases involving newborns 30 days of age or younger undergoing ligation of a patent ductus arteriosus only. The total annual case volume for cardiac surgical cases meeting the above criteria was then computed for each center, and the 22 institutions with codes indicating cardiac surgery in at least 100 discharges per year were analyzed further.

\section{Analysis of Unadjusted Mortality Rates}

For each institution performing at least 100 cardiac surgical cases per year, an overall mortality rate was calculated for the entire caseload. Institutions were ranked in order of increasing mortality from 1 (lowest mortality) to 22 (highest mortality).

\section{Application of the RACHS-1 Method}

The RACHS-1 method can be applied in 2 ways. The simpler approach groups procedures with similar expected short-term mortality rates into 6 predefined risk categories, in which category 1 has the lowest risk for death and category 6 the highest (Table 1), and examines mortality separately within each category. The more complex multivariate method incorporates 4 additional clinical factors: age stratified as 30 days or less, 31 days to 1 year, and 1 year or older; prematurity identified by the presence of appropriate ICD-9-CM diagnosis codes; presence of a major noncardiac structural anomaly in addition to the cardiac defect (eg, tracheoesophageal fistula, cleft lip, or palate); and presence of combinations of cardiac surgical procedures.

Using computer algorithms, we assigned cases to 1 of the 6 risk categories. Unassigned cases, typically those having codes with vague descriptions, such as "revision of procedure," were excluded. Surgical cases with combinations of cardiac procedures performed simultaneously (eg, coarctation of the aorta and ventricular septal defect closure) were placed in the category corre- 
sponding to the single highest-risk procedure. A category-specific mortality rate was calculated for each institution with at least 10 cases in that category to apply the simpler method of adjustment; centers were then ranked in order of increasing mortality. A graphic display of ranks by risk category was constructed for each institution, and graphs were inspected to identify overall patterns.

Application of the more complex method allows computation of an overall risk-adjusted rank for each institution, incorporating nearly the entire institutional caseload into a single measure of performance. To begin, expected mortality rates, adjusting for case mix, were calculated for each center. Risk categories 2, 3, 4, and 6 (no cases were placed in risk category 5 , which contains very few procedures) were used as binary covariates in a logistic regression model predicting mortality, with category 1 as the reference group. The resulting model was then used to calculate the predicted probability of death for each individual case in the data set. The average predicted probability of death for all cases within a particular institution, which was calculated by summing the predicted probabilities for each case and dividing by the total number of cases, represents the expected mortality rate for that center, adjusting for case mix. The observed and expected mortality rates were compared by calculating the standardized mortality ratio (SMR) for each center, which was defined as the observed mortality rate divided by the expected mortality rate. If the observed mortality rate for an institution is higher than expected, meaning that the center performs worse than would be expected given its case mix, the SMR is greater than 1. If the institution's observed mortality rate is lower than expected, indicating better performance than would be anticipated, the SMR is less than 1. Institutions can then be ranked from lowest to highest on the basis of their individual SMR values, providing an assessment of relative performance for the overall caseload.

Similar methodology can be applied for the 4 additional clinical factors to improve the case-mix adjustment further. Binary covariates representing age 30 days or less, age 31 days to 1 year, prematurity, presence of a major noncardiac structural anomaly, and presence of combinations of cardiac surgical procedures were included in the logistic regression model already containing risk category. The resulting model was used to calculate the predicted probability of death for each patient in the data set; the average predicted probability of death for all patients within a particular institution is the expected mortality rate for that center, adjusting for risk category and the other clinical factors. Once again, observed and expected mortality rates for each institution were compared using SMR values, and institutions were ranked according to increasing SMR.

A $95 \%$ confidence interval was calculated for each SMR. ${ }^{10}$ If the confidence interval contains the value 1 , the difference in observed versus expected rates is not considered to be statistically significant.

\section{Results}

In 6 states 7177 cases of surgery for congenital heart disease were identified in children less than 18 years of age. These cases were performed at 109 institutions, with a median annual case volume of 11 (range, 1-587 cases). Of these centers, 22 performed at least 100 cases, accounting for $72.3 \%$ of the total surgical procedures performed. The mor- tality rate for the entire data set was 5.6\%. The 22 large institutions had an overall mortality rate of $4.0 \%$, whereas the 87 smaller institutions had a mortality rate of $9.8 \%$. The range in unadjusted mortality rates for the 22 large centers was $2.5 \%$ to $11.4 \%$, with a median of $5.6 \%$. Using blinded alphabet codes, the ranked mortality rates for each institution are shown in Table 2.

A total of $4318(83.2 \%)$ cases could be placed into 1 of the 6 risk categories; the remaining cases were uncategorizable and are not included in further analyses. Institutionspecific mortality rates for only those cases assigned to a risk category are shown in Table 2; for these cases, the range in unadjusted mortality rates was $1.7 \%$ to $11.0 \%$, with a median of $4.5 \%$.

The caseload for each institution was then stratified by risk category. No cases were identified in category 5; this category was therefore excluded from further analyses. Within each of the remaining risk categories, mortality rates were computed for institutions performing 10 or more cases within that category. All 22 institutions performed at least 10 cases in risk categories 1,2 , and 3; 10 institutions performed 10 or more cases in category 4; and only 3 institutions performed 10 or more cases in category 6 . Institutional mortality rates for each risk category are shown in Figure 1.

Within each risk category, institutions were ranked in order of increasing mortality rate. Very few deaths occurred in risk category 1 , and thus relative institutional performance in this category was not meaningful. Similarly, too few institutions performed procedures in risk category 6 to make informative comparisons. Considerable variation in mortality was observed in categories 2, 3, and 4. In category 2 mortality rates ranged from $0 \%$ to $8.8 \%$ (median, $2.5 \%$ ), whereas rates ranged from $0 \%$ to $20.8 \%$ (median, $4.6 \%$ ) in category 3 and from $5.3 \%$ to $40 \%$ (median, $10.4 \%$ ) in category 4. Graphs of the mortality rate rankings within these 3 risk categories for each institution are shown in Figure 2. Because all 22 institutions are ranked in categories 2 and 3 but only 10 are ranked in category 4, the graph displays the relative ranking for each center, defined as the absolute rank divided by the total number of institutions ranked in that risk category. Thus, for example, the institution receiving rank 11 in risk category 2 would be assigned a relative rank of $11 / 22=0.5$; similarly, the institution receiving rank 5 in risk category 4 would be assigned a relative rank of $5 / 10=0.5$.

Inspection of the 22 sets of rankings revealed overall patterns of some interest for each institution (Figure 3). Twelve institutions have consistent relative rankings for each of the 2 or 3 risk categories in which they are ranked (Figure 3, A). For these institutions, relative performance can be classified as being consistently good, average, or poor. Five institutions seem to have a threshold increase in 


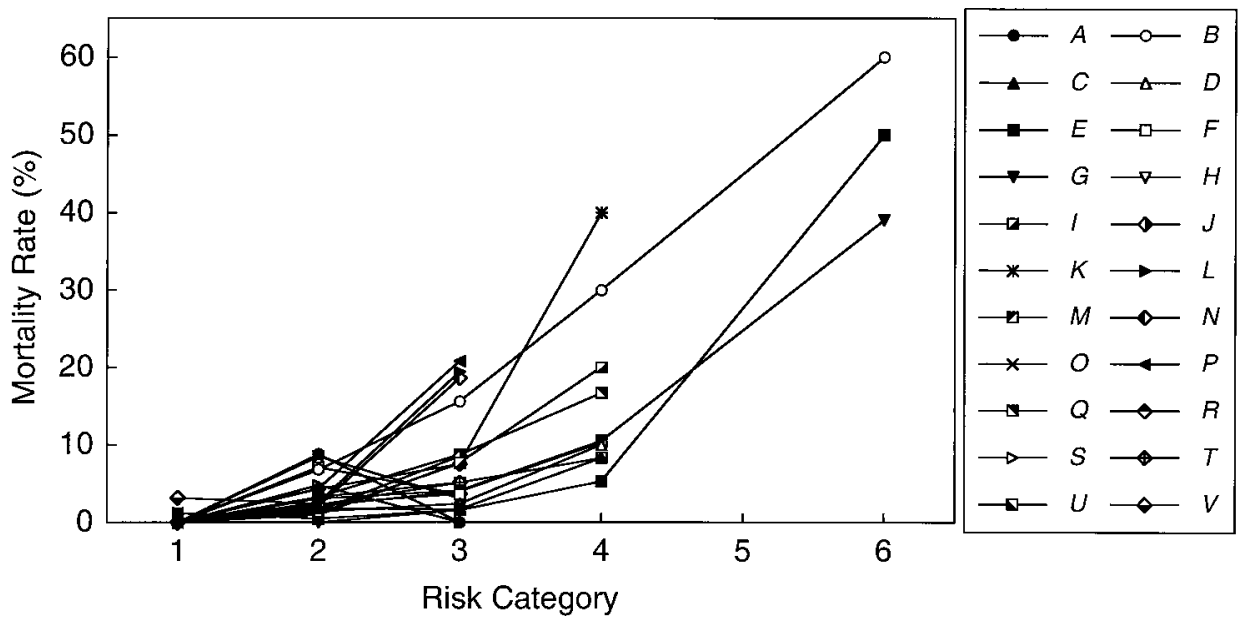

Figure 1. Institution-specific mortality rates for cases in risk categories 1, 2, 3, 4, and 6.

Table 2. Observed and expected mortality rates by institution

\begin{tabular}{|c|c|c|c|c|c|c|c|c|c|c|}
\hline \multirow[b]{2}{*}{ Center } & \multicolumn{2}{|c|}{ All cases } & \multicolumn{2}{|c|}{$\begin{array}{l}\text { All cases in risk } \\
\text { category }\end{array}$} & \multicolumn{2}{|c|}{$\begin{array}{l}\text { Adjusted for risk } \\
\text { category }\end{array}$} & \multicolumn{4}{|c|}{$\begin{array}{l}\text { Adjusted for risk category and additional clinical } \\
\text { factors }\end{array}$} \\
\hline & MR & Rank & MR & Rank & $\begin{array}{l}\text { Expected } \\
\text { MR }\end{array}$ & Rank & $\begin{array}{l}\text { Expected } \\
\text { MR }\end{array}$ & SMR & $95 \% \mathrm{Cl}$ & Rank \\
\hline C & $2.5 \%$ & 1 & $1.9 \%$ & 2 & $4.5 \%$ & 2 & $4.5 \%$ & 0.43 & $(0.09-1.25)$ & 1 \\
\hline D & $3.6 \%$ & 7 & $2.2 \%$ & 4 & $4.8 \%$ & 3 & $4.7 \%$ & 0.48 & $(0.15-1.11)$ & 2 \\
\hline $\mathrm{H}$ & $2.7 \%$ & 3 & $1.7 \%$ & 1 & $4.1 \%$ & 1 & $3.3 \%$ & 0.51 & $(0.10-1.48)$ & 3 \\
\hline$E$ & $2.9 \%$ & 4 & $2.4 \%$ & 5 & $5.0 \%$ & 4 & $4.6 \%$ & 0.53 & $(0.27-0.92)$ & 4 \\
\hline M & $3.7 \%$ & 8 & $3.4 \%$ & 7 & $5.1 \%$ & 6 & $5.3 \%$ & 0.64 & $(0.32-1.15)$ & 5 \\
\hline $\mathrm{F}$ & $2.5 \%$ & 2 & $3.0 \%$ & 6 & $4.7 \%$ & 5 & $4.5 \%$ & 0.67 & $(0.13-1.96)$ & 6 \\
\hline G & $5.5 \%$ & 11 & $5.6 \%$ & 17 & $6.6 \%$ & 9 & $7.0 \%$ & 0.80 & $(0.50-1.23)$ & 7 \\
\hline S & $3.1 \%$ & 5 & $2.0 \%$ & 3 & $2.7 \%$ & 7 & $2.4 \%$ & 0.83 & $(0.09-3.00)$ & 8 \\
\hline U & $6.3 \%$ & 15 & $4.2 \%$ & 9 & $5.2 \%$ & 8 & $4.7 \%$ & 0.89 & $(0.44-1.60)$ & 9 \\
\hline I & $5.6 \%$ & 12 & $4.2 \%$ & 9 & $4.4 \%$ & 10 & $4.5 \%$ & 0.92 & $(0.49-1.58)$ & 10 \\
\hline $\mathrm{T}$ & $4.8 \%$ & 9 & $4.5 \%$ & 11 & $4.1 \%$ & 11 & $4.2 \%$ & 1.06 & $(0.34-2.49)$ & 11 \\
\hline V & $3.4 \%$ & 6 & $3.9 \%$ & 8 & $3.5 \%$ & 12 & $3.4 \%$ & 1.12 & $(0.30-2.86)$ & 12 \\
\hline $\mathrm{R}$ & $6.1 \%$ & 14 & $5.1 \%$ & 13 & $4.5 \%$ & 14 & $4.3 \%$ & 1.20 & $(0.44-2.60)$ & 13 \\
\hline K & $6.4 \%$ & 16 & $5.2 \%$ & 14 & $4.5 \%$ & 13 & $4.3 \%$ & 1.21 & $(0.60-2.16)$ & 14 \\
\hline$J$ & $6.5 \%$ & 17 & $6.2 \%$ & 18 & $4.9 \%$ & 16 & $4.9 \%$ & 1.25 & $(0.67-2.14)$ & 15 \\
\hline 0 & $6.7 \%$ & 18 & $5.5 \%$ & 16 & $4.3 \%$ & 17 & $4.4 \%$ & 1.26 & $(0.70-2.07)$ & 16 \\
\hline $\mathrm{L}$ & $8.8 \%$ & 19 & $7.8 \%$ & 19 & $4.3 \%$ & 19 & $5.3 \%$ & 1.47 & (0.63-2.89) & 17 \\
\hline A & $5.0 \%$ & 10 & $4.6 \%$ & 12 & $3.7 \%$ & 15 & $3.1 \%$ & 1.50 & $(0.40-3.85)$ & 18 \\
\hline 0 & $6.0 \%$ & 13 & $5.4 \%$ & 15 & $3.6 \%$ & 18 & $3.6 \%$ & 1.51 & $(0.55-3.28)$ & 19 \\
\hline $\mathrm{N}$ & $11.4 \%$ & 22 & $10.1 \%$ & 21 & $5.1 \%$ & 20 & $6.5 \%$ & 1.56 & $(0.80-2.72)$ & 20 \\
\hline$P$ & $10.0 \%$ & 20 & $9.8 \%$ & 20 & $3.9 \%$ & 22 & $4.8 \%$ & 2.02 & $(0.92-3.84)$ & 21 \\
\hline B & $10.7 \%$ & 21 & $11.0 \%$ & 22 & $5.2 \%$ & 21 & $5.2 \%$ & 2.11 & $(1.40-3.05)$ & 22 \\
\hline
\end{tabular}

MR, Mortality rate; SMR, standardized mortality ratio (ie, observed MR/expected MR); $C l$, confidence interval.

mortality: as higher-risk procedures are performed, relative rankings increase (Figure 3, B). In all instances worse performance occurs between categories 2 and 3 . The 5 remaining institutions seem to have a threshold decrease in mortality: as higher-risk procedures are performed, relative rankings improve (Figure 3,C). Four centers show worse ranks for category 2 than for categories 3 and 4 , and one has poorer ranks in categories 2 and 3 relative to category 4 .
Expected mortality rates and SMRs for each institution are shown in Table 2 to assess overall institutional performance adjusted for risk category. Ranks are assigned according to increasing SMR.

Expected mortality rates and SMRs adjusting for age, prematurity, presence of a major noncardiac structural anomaly, and combinations of cardiac procedures in addition to risk category are also shown in Table 2 to refine the 


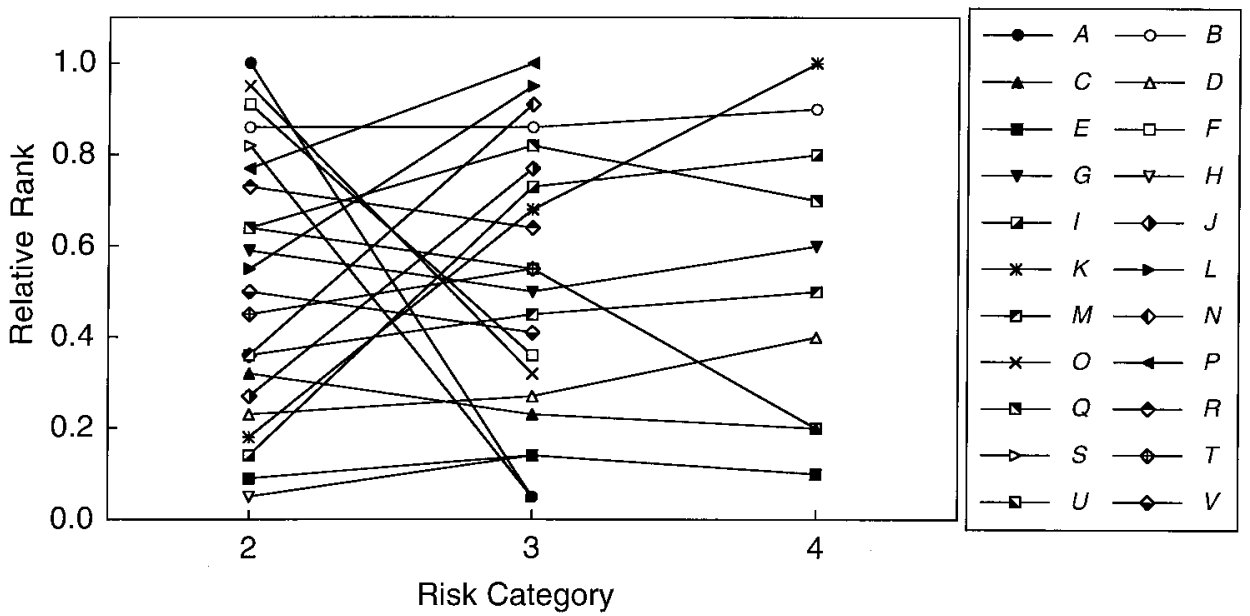

Figure 2. Relative ranks for institution-specific mortality rates in risk categories 2, 3, and 4 . Relative ranks are defined as the absolute rank divided by the total number of institutions ranked in that risk category.

case-mix adjustment further. The rankings displayed only minor changes.

The additional information provided by the more complex multivariate risk-adjustment method can be inferred from inspection of the expected institutional mortality rates in Table 2. Although the mortality rate for the group as a whole is $4.0 \%$, the expected mortality rates at each institution vary nearly 3 -fold, from $2.4 \%$ to $7.0 \%$.

\section{Discussion}

Quality-improvement efforts can be enhanced and stimulated by a clear understanding of how an institution is performing in comparison with other institutions, as well as how its performance is changing over time. In this report we used the newly developed RACHS-1 method to try to understand differences in short-term mortality for congenital heart procedures. We analyzed 1996 hospital discharge data from 22 institutions in 6 states, each performing at least 100 operations for congenital heart disease in children. By limiting the study to centers with larger case volumes, we hoped to reduce volume-dependent effects; volume has been shown to be a determinant of center-specific differences in mortality. ${ }^{1-7}$

Despite the broad diversity of procedures performed at each of the centers, we were able to create risk-adjusted measures and rankings of overall performance for each institution. We applied the RACHS-1 methodology to examine risk category-specific mortality rates and to calculate SMRs, adjusting for risk category and other clinical factors. Although most of the descriptive information could be derived from the simpler method, which adjusted for risk category only, the incorporation of additional clinical factors also explained some of the differences in observed mortality, as evidenced by the changes in expected mortal- ity rates when these additional variables were incorporated into the model.

In addition to the institutional performance rankings listed in Table 2, important insights about mortality differences have come from analysis of the information within each risk category. All of the institutions in our analysis were able to perform procedures in risk category 1 with little, if any, mortality. For higher-risk procedures, several distinct patterns emerged. Some institutions displayed a consistent relative performance across risk categories. For institutions performing at better than average levels, focus should be placed on improving outcomes for higher-risk procedures, especially those in categories 4 and 6 , in which overall mortality rates remain high. Institutions performing considerably worse than average for all risk categories may need to consider global, rather than incremental, programmatic changes. Several institutions showed worsening performance for higher-risk procedures. These patterns may suggest that institutions should consider targeted referral to centers with better performance or other measures to improve performance for higher-risk procedures. Some institutions displayed an interesting pattern of poorer performance for lower-risk than for higher-risk procedures. For programs with this pattern, careful intrainstitutional assessment should be made of possible systematic differences (eg, assignment of certain types of cases to specific surgeons and location of postoperative care) in the way that simpler cases are handled in comparison with more complex ones. In any case the finding of increasing or decreasing risk-adjusted performance as complexity increases should prompt focused inquiries within institutions about potential explanatory factors.

Although analyses such as these should prompt inquiry, definitive conclusions about quality of care at individual 

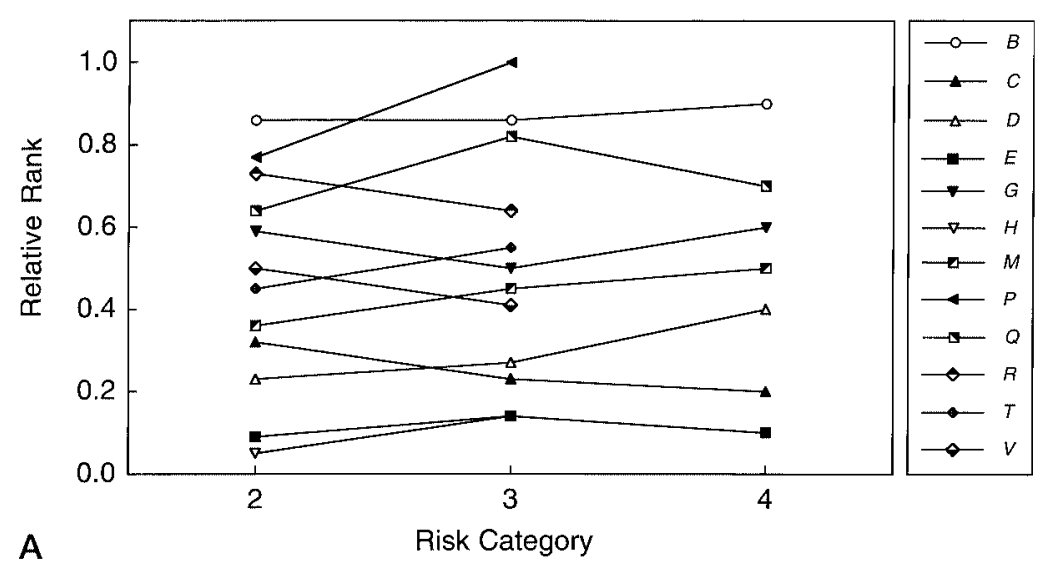

A
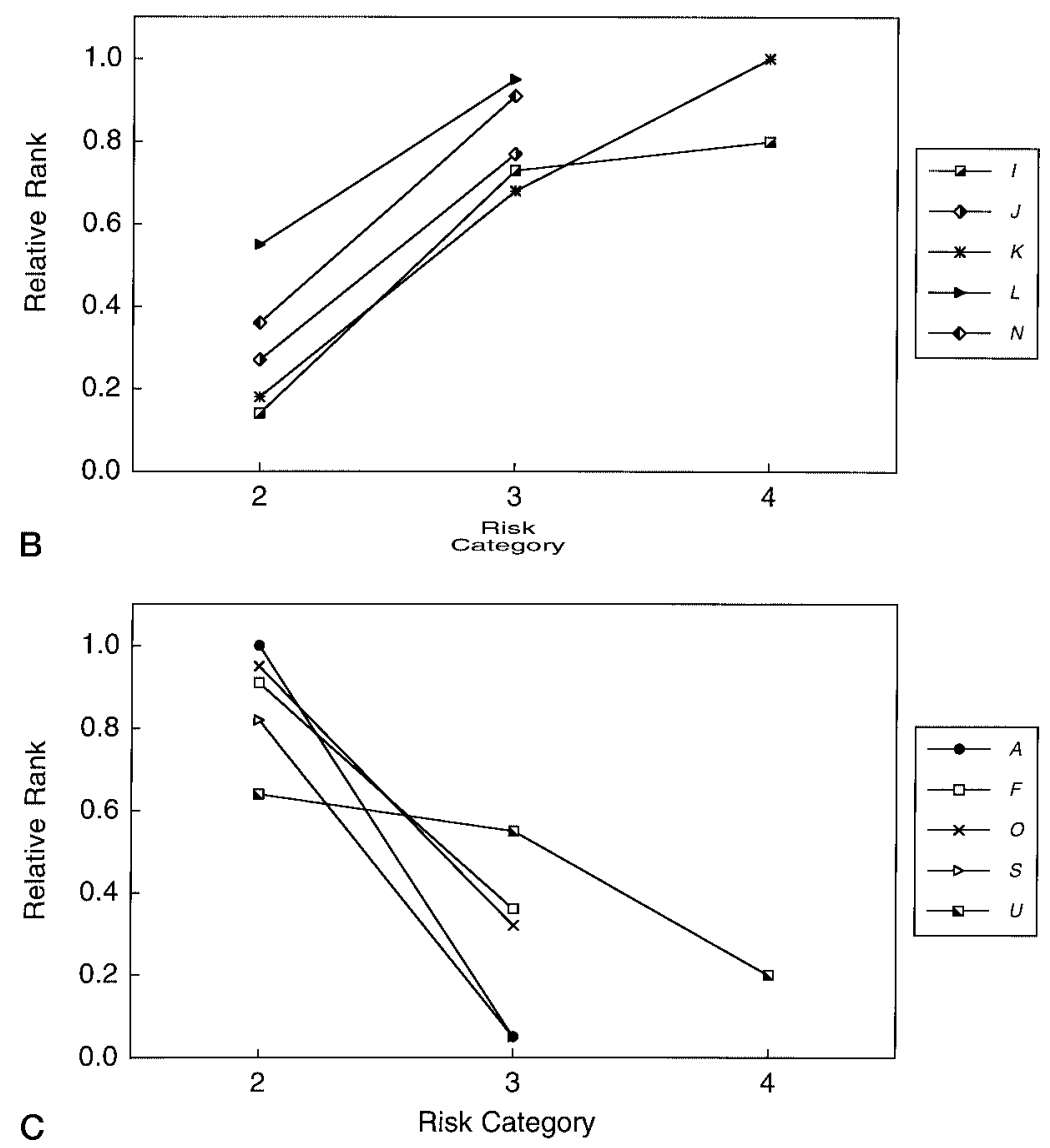

Figure 3. Relative ranks for institution-specific mortality rates in risk categories 2, 3, and 4 separated into institutions with nearly equivalent rankings in all risk categories (A), institutions with a threshold increase in mortality as higher-risk procedures are performed (B), and institutions with a threshold decrease in mortality as higher-risk procedures are performed (C).

centers should not be made from this type of investigation. Data analyses should be considered exploratory, given the many possible competing explanations for higher-than-expected death rates at some institutions, including data-coding errors, case-mix differences insufficiently accounted for with the RACHS-1 method, and chance variability, espe- cially for the analysis of a single time period. Nearly all of the differences demonstrated did not reach statistical significance in this single calendar year. Furthermore, inaccuracies in the data may be present because hospital discharge data are collected primarily to support billing and are verified by various statewide agencies charged with this pur- 
pose and not by investigators. We suspect that procedures might be more accurately coded than diagnoses as a part of the billing process, but this has not been tested. Similarly, despite its importance, in-hospital mortality is only one of the clinically important end points that should be of interest to program directors. Relative performance with respect to late mortality and functional and neurologic outcomes, as examples, would also be of obvious interest for efforts at quality improvement. Such analyses would require specific data collection and the development of risk-adjustment tools beyond RACHS-1. Better data sets would clearly improve the accuracy of overall assessments in general.

\section{Summary}

The primary purpose of this study was to test whether the newly created RACHS- 1 method could improve understanding of institution-specific mortality for congenital heart surgery. As a first step, use of the method seemed successful: the variation in both unadjusted $(2.5 \%-11.4 \%)$ and expected (2.4\%-7.0\%) mortality rates at the 22 institutions reconfirms the importance of risk-adjustment techniques in assessing institutional performance. In addition, despite limiting analyses to institutions performing larger caseloads and using risk adjustment, some institutions performed substantially better or worse than expected. Although most differences were not statistically significant in this single calendar year, interesting possibilities for targeted improvements may come from a center-by-center analysis of performance in different risk categories. In combination, these observations would seem to confirm the need for risk adjustment when comparing short-term mortality after surgery for congenital heart disease, as well as supporting the potential utility of the RACHS- 1 method.

We thank Ms Deborah Quigley and Mr Gary Piercey for their programming and administrative assistance with this project.

\section{References}

1. Gutgesell HP, Massaro TA, Kron IL. The arterial switch operation for transposition of the great arteries in a consortium of university hospitals. Am J Cardiol. 1994;74:959-60.

2. Gutgesell HP, Massaro TA. Management of hypoplastic left heart syndrome in a consortium of university hospitals. Am J Cardiol. 1995;76:809-11.

3. Jenkins KJ, Newburger JW, Lock JE, Davis RB, Coffman GA, Iezzoni LI. In-hospital mortality for surgical repair of congenital heart defects: preliminary observations of variation by hospital caseload. Pediatrics. 1995;95:323-30.

4. Hannan EL, Racz M, Kavey RE, Quaegebeur JM, Williams R. Pediatric cardiac surgery: the effect of hospital and surgeon volume on in-hospital mortality. Pediatrics. 1998;101:963-9.

5. Sollano JA, Gelijns AC, Moskowitz AJ, Heitjan DF, Cullinane S, Saha $\mathrm{T}$, et al. Volume-outcome relationships in cardiovascular operations: New York state, 1990-1995. J Thorac Cardiovasc Surg. 1999;117: 419-30.

6. Stark J, Gallivan S, Lovegrove J, Hamilton JRL, Monro JL, Pollock JCS, et al. Mortality rates after surgery for congenital heart defects in children and surgeon's performance. Lancet. 2000;355:1004-7.

7. Jenkins KJ, Gauvreau K. Effect of institutional volume on mortality for congenital heart surgery [abstract]. Circulation. 2000;102(Suppl II):\#18.

8. Jenkins KJ, Gauvreau K, Newburger JW, Spray T, Moller JH, Iezzoni L. Consensus-based method for risk adjustment for surgery for congenital heart disease. J Thorac Cardiovasc Surg. 2002;123:110-8.

9. International classification of diseases, 9th revision. 4th ed. Clinical modification. Los Angeles: Practice Management Information Corp; 1992.

10. Breslow NE, Day NE. Statistical methods in cancer research. Vol II. The design and analysis of cohort studies. New York: Oxford University Press; 1987.

\section{Availability of Journal back issues}

As a service to our subscribers, copies of back issues of The Journal of Thoracic and Cardiovascular Surgery for the preceding 5 years are maintained and are available for purchase from Mosby until inventory is depleted. Please write to Mosby, Subscription Customer Service, 6277 Sea Harbor Dr, Orlando, FL 32877, or call $800-654-2452$ or $407-345-4000$ for information on availability of particular issues and prices. 